\title{
Radiación ionizante recibida por pruebas de radiología intervencionista durante el tratamiento con quimioterapia intraarterial en pacientes afectos de osteosarcoma
}

\section{Ionizing radiation received by patients with osteosarcoma during intra-arterial chemotherapy treatment}

doi.org/10.23938/ASSN.0009

\author{
N. Aznárez-Sanado ${ }^{1}$, M. Aznárez-Sanado ${ }^{2}$, L. Sierrasesúmaga ${ }^{1}$, J.I. Bilbao ${ }^{3}$, \\ J.M. Martí-Climent ${ }^{4}$ A. Patiño-García ${ }^{1}$
}

\section{RESUMEN}

Fundamento. Los pacientes afectos de osteosarcoma reciben tratamiento con quimioterapia administrada por vía intraarterial (QTia) ${ }^{3}$ directamente al tumor y son expuestos a radiación ionizante durante el mismo. Los pacientes pediátricos son especialmente vulnerables a esta exposición.

Material y métodos. Se registró la cantidad de radiación ionizante recibida por 16 pacientes pediátricos afectos de osteosarcoma durante la administración de QTia en la Clínica Universidad de Navarra.

Resultados. La mediana de radiación total recibida fue de $33,4 \mathrm{~Gy} \cdot \mathrm{cm}^{2}$ (IQR $43,33 \mathrm{~Gy} \cdot \mathrm{cm}^{2}$ ) y la mediana de número de pruebas por paciente de 10 (IQR: 6,5).

Conclusión. El estudio resalta la importancia de cuantificar la radiación recibida por un grupo de niños y adolescentes afectos de osteosarcoma durante el tratamiento con QTia ya que no conviene olvidar los potenciales efectos adversos a largo plazo de esta exposición. Actualmente, no existen estudios previos que aporten datos acerca de la cantidad de radiación ionizante recibida a través de este procedimiento.

Palabras clave: Radiación ionizante. Radiología intervencionista. Pediatría. Osteosarcoma.

\begin{abstract}
Background. Osteosarcoma paediatric patients are usually treated with intra-arterial chemotherapy (QTia) which is administered directly to the tumour. This procedure exposes patients to ionizing radiation. Paediatric patients are especially sensitive to this exposure.
\end{abstract}

Methods. The total amount of ionizing radiation received from QTia administration was quantified in a group of 16 osteosarcoma paediatric patients from the Clínica Universidad de Navarra.

Results. The median of the total radiation received per patient was $33.4 \mathrm{~Gy} \cdot \mathrm{cm}^{2}$ (IQR: $43.33 \mathrm{~Gy} \cdot \mathrm{cm}^{2}$ ), and the median number of procedures performed per subject was 10 (IQR: 6.5 ).

Conclusions. The study highlights the importance of quantifying the radiation received by a group of children and adolescents affected by osteosarcoma during treatment with QTia. Long-term side effects of this radiation should be considered in paediatric patients. $\mathrm{Cu}$ rrently, there are no previous studies that provide data of the amount of ionizing radiation received through this procedure.

Keywords: Ionizing radiation. Interventional radiology. Paediatrics. Osteosarcoma.
1. Departamento de Pediatría. Clínica Universidad de Navarra. Pamplona. Navarra.

2. Departamento de Teoría y Métodos de Investigación Educativa y Psicológica. Facultad de Educación y Psicología. Universidad de Navarra. Pamplona. Navarra.

3. Departamento de Radiología. Responsable de la Unidad de Radiología Vascular e Intervencionista. Clínica Universidad de Navarra. Pamplona. Navarra.

4. Responsable de la Unidad de Radiofísica. Clínica Universidad de Navarra. Pamplona. Navarra.

\section{Correspondencia:}

Nerea Aznárez Sanado

Departamento de Pediatría

Clínica Universidad de Navarra

Avenida Pío XII, 36

31008 Pamplona

E-mail: nerea265@hotmail.com

Recepción: 30/06/2016

Aceptación provisional: 11/11/2016

Aceptación definitiva: 22/12/2016 


\section{INTRODUCCIÓN}

El uso de pruebas de imagen en procedimientos de radiología intervencionista está aumentado en pacientes pediátricos ${ }^{1}$. Además, la complejidad y la duración de cada procedimiento son cada vez mayores, por lo que la dosis de radiación total recibida por los pacientes puede verse incrementada.

La edad es un factor fundamental a la hora de optimizar la exposición a la radiación ionizante y los pacientes en edad pediátrica deben ser tratados con más cuidado aún que los adultos. Según la definición de la OMS, los pacientes pediátricos se clasifican en: recién nacido (hasta los 28 días de vida), lactante/niño de corta edad (hasta los 2 años de vida), niño preescolar (2 a 5 años), niño escolar (6 a 11 años) y adolescencia (de los 11 años a los 18 años). Un niño y un adolescente tienen mayor riesgo de desarrollar cáncer recibiendo la misma dosis de radiación que un adulto, y esto es debido a diversos factores. Por un lado, tienen mayor esperanza de vida, por lo que tienen más tiempo para presentar efectos secundarios a la radiación recibida. Además, presentan mayor sensibilidad biológica con la misma dosis, ya que poseen mayor cantidad de tejido en proliferación ${ }^{1}$. Por último, es importante resaltar que los equipos de radiología están mínimamente adaptados a pacientes pediátricos ${ }^{2}$.

El osteosarcoma es un tumor maligno derivado de células mesenquimales primitivas y corresponde al $2-4 \%$ de todas las neoplasias pediátricas ${ }^{3}$. Su distribución es bimodal, con picos durante la adolescencia y en adultos mayores de 65 años. Normalmente se desarrollan en huesos largos, siendo las localizaciones más frecuentes el fémur distal, la tibia proximal y el húmero proximal. Por otro lado, las metástasis pulmonares son las más frecuentes.

El tratamiento del osteosarcoma comprende cirugía y quimioterapia pre y postoperatoria. En la Clínica Universidad de Navarra (CUN), la quimioterapia preoperatoria se administra preferentemente por vía intraarterial ${ }^{3}$. Las pruebas de radiología intervencionista se utilizan para administrar quimioterapia intraarterial (QTia) directamente a la lesión tumoral primaria ${ }^{4}$, introduciendo los fármacos en la arteria que suministra sangre al tumor. Se hace uso de la angiografía, una técnica de imagen que emite radiación ionizante mientras guía al radiólogo durante la administración del contraste radioopaco por vía intravascular.

En nuestro centro, la administración de QTia mediante angiografía es un pilar fundamental en los pacientes afectos de osteosarcoma. Actualmente, no existen estudios que aporten datos acerca de la cantidad de radiación ionizante recibida a través de este procedimiento. Por este motivo, el objetivo del presente estudio fue cuantificar la radiación total recibida por una serie de niños y adolescentes afectos de osteosarcoma durante los procedimientos de radiología intervencionista que permiten la administración de la QTia.

\section{PACIENTES Y MÉTODOS}

Se registró, de forma retrospectiva, la cantidad de radiación ionizante procedente de las pruebas de radiología intervencionista recibida por 70 pacientes afectos de osteosarcoma, desde el diagnóstico de su enfermedad hasta un máximo de 10 años de seguimiento. Todos los pacientes fueron tratados en la Unidad de Oncopediatría de la CUN entre los años 1993 y 2015. Todos los participantes en el proyecto dieron su consentimiento para participar en el mismo y el proyecto fue aprobado por el Comité de Ética e Investigación clínica de la CUN.

Una de las medidas más empleadas en múltiples estudios para expresar el impacto de la radiación por cada prueba de imagen es la dosis efectiva. Sin embargo, en el presente estudio, se consideró que esta magnitud presenta ciertos inconvenientes cuando se estudia una muestra de pacientes en edad pediátrica y en su adolescencia, por lo que se decidió no utilizarla. La radiación total recibida por los pacientes fue cuantificada a través de la dosis absorbida en superficie, tomando como referen- 
cia la publicación 121 de la International Commission on Radiological Protection ${ }^{1}$. En las pruebas de radiología intervencionista, la radiación se expresa en forma de producto dosis-área (PDA), expresado en unidades $\mathrm{Gy} \cdot \mathrm{cm}^{2}$; se suele indicar además el tiempo de fluoroscopia y el número total de imágenes adquiridas ${ }^{5}$.

Tras revisar el PDA en cada prueba de imagen, se objetivó que no había sido registrado para las pruebas de radiología intervencionista realizadas antes del año 2003. La variabilidad inter-prueba era alta, por lo que no fue posible obtener un promedio de PDA por grupo de edad. Por este motivo, en el presente estudio se incluyeron únicamente los datos de aquellos pacientes afectos de osteosarcoma para los que estaba registrado el $70 \%$ o más de los valores de PDA en todos los procedimientos de angiografía realizados.

\section{Técnica utilizada para realizar la QTia}

Aunque el tratamiento de los pacientes con osteosarcoma en la CUN se individualiza en función de la evolución del paciente, generalmente se realizan entre 3 y 4 ciclos de tratamiento con QTia prequirúrgica. Cada ciclo de tratamiento consta de 3 sesiones realizadas en 3 días consecutivos, que se repiten cada 21 días. En cada una de ellas, se administra QTia directa a la lesión.

Cada procedimiento se practica previa sedación del paciente y tras punción, con técnica de Seldinger, de las arterias femorales comunes, alternando entre ambas. Tras colocar el extremo de un catéter $4 \mathrm{~F}$ en la arteria principal más próxima a la lesión (p.ej. la poplítea en una lesión de rodilla) se realiza una arteriografía que permite conocer el grado de vascularización de la lesión y realizar un plano vascular de los vasos aferentes a la lesión. Con un microcatéter (2,7 F) se accede a cada uno de los vasos y se introduce la medicación en forma de "bolus" durante 5-15 minutos. Una vez finalizado el procedimiento, se extrae el material angiográfico y se comprime manualmente la femoral. Aunque es muy va- riable en función del tipo de lesión, en cada sesión de QTia se realizan unas 2-3 tomas de imágenes digitales con 30 imágenes en cada toma (durante 10 segundos se realizan 3 imágenes por segundo).

\section{Análisis estadístico}

Una vez obtenidos todos los datos de radiación, se procedió al análisis de los mismos. Para cada paciente, se estimó la cantidad total de radiación ionizante recibida a través de los procedimientos de radiología intervencionista, así como el número de pruebas realizadas en cada uno de ellos. Asimismo, se estimó la cantidad promedio de radiación ionizante recibida en cada procedimiento de radiología intervencionista dividiendo la radiación total recibida entre el número de pruebas realizadas. Debido a que el conjunto de datos correspondientes a la cantidad total de radiación recibida por paciente no seguían una distribución normal (test de Shapiro-Wilk, $\mathrm{p}<0.001$ ), se realizó un análisis descriptivo de los datos de grupo expresando los valores obtenidos a través de la mediana y el rango intercuartílico (IQR). Finalmente, se realizó un análisis de regresión lineal (ANOVA de regresión) para evaluar el grado de relación existente entre la radiación total recibida y el número total de procedimientos llevados a cabo en cada paciente.

Aunque el concepto de adolescente según la OMS abarca hasta los 18 años, en España y en concreto en Navarra, la atención pediátrica se realiza hasta el final de los 14 años. Debido a este corte de edad, se consideró de interés aportar datos de radiación incluyendo únicamente a los pacientes menores de 15 años del estudio. Asimismo, se realizaron comparaciones entre la cantidad total de radiación recibida por pacientes menores de 15 años y la recibida por aquellos de edades comprendidas entre 15 y 18 años. El análisis estadístico empleado para esta comparación fue Mann-Whitney. $\mathrm{El}$ análisis de regresión lineal también fue repetido incluyendo únicamente a este grupo de edad. 


\section{RESULTADOS}

Tras la revisión de la historia clínica de los 70 pacientes diagnosticados de osteosarcoma, se identificaron un total de 16 pacientes menores de 18 años donde estaba registrado el $70 \%$ o más de los PDA administrados en cada uno de los procedimientos de radiología intervencionista realiza- dos (Tabla 1). El resto de pacientes fueron excluidos del estudio. La mediana de edad al diagnóstico en este grupo de pacientes fue 12 años (IQR 6,3 años) y la mediana de tiempo de seguimiento fue 27,5 meses (IQR 34,5 meses). Es importante destacar que la mediana de peso y talla de los pacientes del estudio fue 45,8 Kg (IQR 18,2 kg) y 159 cm (IQR 21,8 cm) respectivamente.

Tabla 1. Características clínicas de los pacientes del presente estudio.

\begin{tabular}{|c|c|c|c|c|c|c|c|c|c|c|}
\hline Id & $\begin{array}{l}\text { Género } \\
\text { (mujer, } \\
\text { varón) }\end{array}$ & $\begin{array}{l}\text { Edad al } \\
\text { diagnóstico } \\
\text { (años) }\end{array}$ & $\begin{array}{r}\text { Peso } \\
\text { (kg) }\end{array}$ & $\begin{array}{l}\text { Talla } \\
(\mathrm{cm})\end{array}$ & $\begin{array}{l}\text { Localiza- } \\
\text { ción }\end{array}$ & $\begin{array}{l}\text { Metás- } \\
\text { tasis }\end{array}$ & $\begin{array}{c}\text { Recidiva } \\
\text { local }\end{array}$ & $\begin{array}{l}\text { Estado } \\
\text { (vivo, } \\
\text { fallecido) }\end{array}$ & $\begin{array}{l}\text { Meses de } \\
\text { seguimiento } \\
\text { tras el } \\
\text { diagnóstico }\end{array}$ & Incidencias \\
\hline 1 & M & 17 & 53 & 176 & $\begin{array}{l}\text { Fémur } \\
\text { distal }\end{array}$ & No & No & V & 56 & Ninguna \\
\hline 2 & V & 16 & 55 & 159 & Húmero & Sí & No & $\mathrm{F}$ & 8 & $\begin{array}{l}\text { Retraso de } 6 \text { meses en diagnóstico } \\
\text { en otro centro. Múltiples metásta- } \\
\text { sis pulmonares bilaterales. Afecta- } \\
\text { ción de hueso y tejido blando. }\end{array}$ \\
\hline 3 & M & 15 & 44 & 165 & $\begin{array}{c}\text { Peroné } \\
\text { distal }\end{array}$ & Sí & No & $\mathrm{F}$ & 13 & $\begin{array}{l}\text { Múltiples metástasis pulmonares } \\
\text { bilaterales. Afectación de hueso } \\
\text { y tejido blando. Derivado desde } \\
\text { otro país. }\end{array}$ \\
\hline 4 & $\mathrm{~V}$ & 9 & 47 & 155 & Húmero & Sí & Sí & $\mathrm{F}$ & 31 & $\begin{array}{l}\text { Diagnosticado y tratado al inicio } \\
\text { como sarcoma de Ewing. Diagnós- } \\
\text { tico final: osteosarcoma. Recidiva } \\
\text { local. Múltiples metástasis pulmo- } \\
\text { nares bilaterales. }\end{array}$ \\
\hline 5 & $\mathrm{~V}$ & 12 & 38 & 159 & Fémur & No & No & $\mathrm{V}$ & 12 & Ninguna. \\
\hline 6 & V & 10 & 41 & 151 & $\begin{array}{c}\text { Tibia } \\
\text { proximal }\end{array}$ & No & Sí & $\mathrm{F}$ & 37 & Ninguna. \\
\hline 7 & V & 14 & 68,8 & 182 & $\begin{array}{r}\text { Fémur } \\
\text { distal }\end{array}$ & Sí & No & $\mathrm{F}$ & 7 & $\begin{array}{l}\text { Múltiples metástasis pulmonares } \\
\text { bilaterales al diagnóstico }\end{array}$ \\
\hline 8 & V & 16 & 73,4 & 172 & $\begin{array}{l}\text { Fémur } \\
\text { distal }\end{array}$ & No & No & V & 62 & Ninguna. \\
\hline 9 & M & 17 & 72 & 181 & $\begin{array}{l}\text { Fémur } \\
\text { distal }\end{array}$ & Sí & Sí & $\mathrm{F}$ & 37 & $\begin{array}{l}\text { Retraso de } 9 \text { meses en diagnóstico } \\
\text { en otro centro. Afectación vascu- } \\
\text { lar y neural del tumor primario. }\end{array}$ \\
\hline 10 & V & 16 & 72,6 & 174 & $\begin{array}{c}\text { Tibia } \\
\text { proximal }\end{array}$ & Sí & No & V & 50 & Ninguna \\
\hline 11 & V & 5 & 29 & 121 & Fémur & Sí & Sí & V & 24 & $\begin{array}{l}\text { Retraso de } 6 \text { meses en diagnósti- } \\
\text { co en otro país. Múltiples metás- } \\
\text { tasis pulmonares al diagnóstico. }\end{array}$ \\
\hline 12 & M & 12 & 52 & 163 & Fémur & Sí & No & $\mathrm{F}$ & 8 & $\begin{array}{l}\text { Múltiples metástasis pulmonares } \\
\text { al diagnóstico. }\end{array}$ \\
\hline 13 & V & 9 & 31,8 & 140 & $\begin{array}{c}\text { Tibia } \\
\text { proximal }\end{array}$ & Sí & No & V & 48 & Ninguna. \\
\hline 14 & V & 11 & 44,5 & 151,1 & $\begin{array}{l}\text { Tibia } \\
\text { distal }\end{array}$ & No & No & V & 22 & Ninguna. \\
\hline 15 & M & 2 & 11,8 & 84 & $\begin{array}{l}\text { Fémur } \\
\text { completo }\end{array}$ & Sí & No & $\mathrm{F}$ & 14 & $\begin{array}{l}\text { Edad del diagnóstico y tamaño } \\
\text { del tumor primario. }\end{array}$ \\
\hline 16 & M & 10,5 & 43,7 & 150 & $\begin{array}{c}\text { Tibia } \\
\text { proximal }\end{array}$ & No & No & V & 47 & Ninguna. \\
\hline
\end{tabular}


El número de procedimientos, los tiempos medios de radioscopia y la cantidad total de radiación ionizante recibida a través de los procedimientos de radiología intervencionista en este grupo de 16 pacientes se encuentran descritos en la tabla 2.

Tabla 2. Dosis de radiación, tiempos de radioscopia y número total de procedimientos correspondientes a las pruebas de radiología intervencionista.

\begin{tabular}{cccccccc}
\hline $\begin{array}{c}\text { Pacien- } \\
\text { tes del } \\
\text { estudio }\end{array}$ & $\begin{array}{c}\text { Número de } \\
\text { procedimien- } \\
\text { tos realizados } \\
\text { en total }\end{array}$ & $\begin{array}{c}\text { Número de } \\
\text { procedimien- } \\
\text { tos en los que } \\
\text { está registrado } \\
\text { el PDA }\end{array}$ & $\begin{array}{c}\text { Radiación } \\
\text { total recibida } \\
\text { (PDA, Gy·cm) }\end{array}$ & $\begin{array}{c}\text { Promedio } \\
\text { radiación } \\
\text { (Gy·cm)/ } \\
\text { procedi- } \\
\text { miento }\end{array}$ & $\begin{array}{c}\text { Desviación } \\
\text { típica (SD) } \\
\text { radiación reci- } \\
\text { bida (Gy.cm) }\end{array}$ & $\begin{array}{c}\text { Promedio } \\
\text { tiempo de } \\
\text { radioscopia } \\
\text { (min)/procedi- } \\
\text { miento }\end{array}$ & $\begin{array}{c}\text { Desviación } \\
\text { típica (SD) } \\
\text { tiempo de } \\
\text { radioscopia }\end{array}$ \\
\hline $\mathbf{1}$ & 10 & 10 & 46,65 & 4,66 & 5,76 & 74,9 & 13,0 \\
\hline $\mathbf{2}$ & 13 & 13 & 60,55 & 4,66 & 2,65 & 67,2 & 21,2 \\
\hline $\mathbf{3}$ & 9 & 8 & 11,45 & 1,43 & 1,46 & 70,9 & 9,2 \\
\hline $\mathbf{4}$ & 5 & 4 & 11,03 & 2,76 & 1,61 & 61,9 & 22,4 \\
\hline $\mathbf{5}$ & 11 & 10 & 35,62 & 3,56 & 2,46 & 63,9 & 30,8 \\
\hline $\mathbf{6}$ & 18 & 13 & 42,45 & 3,27 & 4,06 & 77,1 & 22,8 \\
\hline $\mathbf{7}$ & 9 & 7 & 29,06 & 4,15 & 4,26 & 65,8 & 12,7 \\
\hline $\mathbf{8}$ & 11 & 10 & 31,08 & 3,11 & 4,16 & 58,7 & 33,9 \\
\hline $\mathbf{9}$ & 18 & 16 & 226,66 & 14,17 & 29,68 & 82,7 & 23,2 \\
\hline $\mathbf{1 0}$ & 31 & 26 & 341,86 & 13,15 & 17,26 & 80,3 & 28,5 \\
\hline $\mathbf{1 1}$ & 29 & 28 & 53,46 & 1,91 & 1,26 & 66,9 & 21,1 \\
\hline $\mathbf{1 2}$ & 11 & 9 & 21,27 & 2,36 & 2,45 & 54,9 & 20,4 \\
\hline $\mathbf{1 3}$ & 4 & 4 & 2,63 & 0,66 & 0,31 & 73,5 & 16,3 \\
\hline $\mathbf{1 4}$ & 11 & 8 & 9,52 & 1,19 & 0,86 & 93,1 & 17,3 \\
\hline $\mathbf{1 5}$ & 18 & 15 & 58,31 & 3,89 & 11,39 & 83,7 & 23,2 \\
\hline $\mathbf{1 6}$ & 8 & 6 & 65,7 & 1,09 & 0,58 & 82,3 & 14,5 \\
\hline & & & & & & & \\
\hline
\end{tabular}

Tras cuantificar la radiación recibida por el grupo de pacientes evaluado a través de las pruebas de radiología intervencionista, se obtuvo una mediana de radiación total de $33,4 \mathrm{~Gy} \cdot \mathrm{cm}^{2}$ (IQR 43,33 $\mathrm{Gy} \cdot \mathrm{cm}^{2}$ ), con una mediana de número de pruebas realizadas de 10 (IQR 6,5). Posteriormente, se determinó, para cada sujeto, la cantidad de radiación promedio recibida en una única sesión, siendo la mediana de radiación promedio del grupo de $3,1 \mathrm{~Gy} \cdot \mathrm{cm}^{2}$ (IQR 2,5 $\mathrm{Gy} \cdot \mathrm{cm}^{2}$ ).

Incluyendo en el análisis de regresión lineal a todos los pacientes menores de 18 años, se obtuvo un valor de $R^{2}=0,459$ (ANOVA de regresión, $\mathrm{p}=0,004$ ). Analizando los diagramas de dispersión y el vector de residuales, se encontró que los datos de 3 pacientes eran outliers (Fig. 1a). Se objetivó que en dos de ellos la radiación recibida era mayor a la esperada en función del número de procedimientos realizados. Tras el análisis de su historia clínica se constató que en uno de los casos la dificultad del procedimiento era mayor debido a la gran vascularización del tumor primario y en el segundo a que las dosis registradas comprendían no sólo la radiación administrada al tumor primario, sino también a las lesiones pulmonares metastásicas, donde el procedimiento es mucho más complejo. En un tercer caso, las dosis eran menores de las esperadas; esto se debe a su menor edad y a que el procedimiento era más sencillo. Excluyendo del análisis los datos de estos 3 pacientes, se obtuvo un valor de 


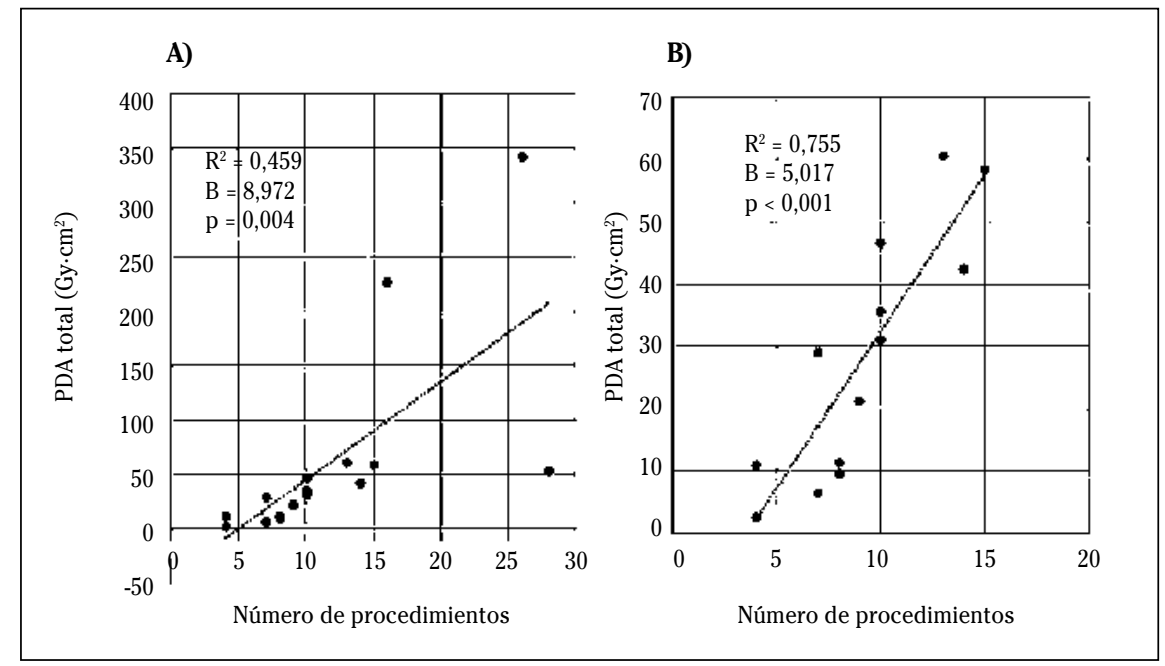

Figura 1. Análisis de regresión lineal entre el número de procesos de radiología intervencionista y la dosis total recibida a) incluyendo a todos los pacientes menores de 18 años y b) excluyendo a los pacientes que resultaron valores extremos

$\mathrm{R}^{2}=0,755$ (ANOVA de regresión, $\mathrm{p}<0,001$ ) (Fig. 1b). En este caso, el número de procedimientos recibidos es capaz de explicar gran parte de la variabilidad observada en el total de radiación recibida por cada paciente, y por lo tanto se puede considerar como un buen predictor de la misma.

Por otro lado, se detectó una gran variabilidad en la radiación inter-prueba en los 16 pacientes del estudio. Aunque la mediana de radiación promedio obtenida fue de $3,1 \mathrm{~Gy} \cdot \mathrm{cm}^{2}$, se llegó a registrar un valor máximo de $123,58 \mathrm{~Gy} \cdot \mathrm{cm}^{2}$ en una única sesión. Este valor fue 4.120 veces superior respecto al mínimo registrado en otro paciente del mismo grupo $\left(0,03 \mathrm{~Gy} \cdot \mathrm{cm}^{2}\right)$. Por otro lado, también se registraron variaciones importantes en un mismo paciente. En uno de los pacientes, se registró un valor máximo de radiación 172 veces superior al mínimo registrado en otro procedimiento realizado durante el mismo año.

Como se ha comentado previamente, debido al corte de edad pediátrica de nuestra comunidad, se consideró de interés realizar un nuevo análisis de los datos centrándonos únicamente en los pacientes menores de 15 años. La mediana de radiación total en este grupo fue de $25,17 \mathrm{~Gy} \cdot \mathrm{cm}^{2}$
(IQR $30,8 \mathrm{~Gy} \cdot \mathrm{cm}^{2}$ ). Comparando estos resultados con los obtenidos en el grupo de edad de 15 a menores de 18 años (53,6 mGy $\mathrm{cm}^{2}$, IQR 150,16 $\left.\mathrm{mGy} \cdot \mathrm{cm} 2\right)$, no se observaron diferencias significativas $(\mathrm{p}=0,051)$. Incluyendo en el análisis de regresión lineal únicamente a los menores de 15 años (en este caso no se detectaron outliers), se obtuvo un valor de $\mathrm{R}^{2}=0,648$ (ANOVA de regresión, $\mathrm{p}=0,005)$. Se considera que en este grupo de edad el número de procedimientos sigue siendo un buen predictor del total de radiación recibida por los pacientes.

\section{DISCUSIÓN}

El objetivo del tratamiento con quimioterapia administrada por vía intraarterial es aumentar la concentración del fármaco en la localización del tumor para incrementar la necrosis del tejido tumoral, reduciendo así la incidencia de recidivas locales y haciendo posible la resección completa de la lesión en mayor número de $\operatorname{casos}^{6}$.

A falta de datos previos, el presente estudio cuantifica de manera objetiva la cantidad de radiación ionizante que los pacientes en edad adolescente afectos de os- 
teosarcoma reciben a través de pruebas de radiología intervencionista. Sin embargo, el tamaño de la muestra es reducido $(\mathrm{N}=16)$, lo que supone una de las principales limitaciones del presente estudio. Además, cabe resaltar que la serie de pacientes incluidos en este estudio no es homogénea en cuanto a la presencia de metástasis y/o la recidiva local del tumor primario, debido a las características individuales de cada sujeto. Esto ha ocasionado que algunos pacientes reciban un mayor número de tratamientos con quimioterapia intraarterial, ya que los ciclos debieron ser repetidos tras la recidiva local. A pesar de estas limitaciones, el presente estudio proporciona dosis de radiación promedio, así como datos acerca de la variabilidad intra- e inter- paciente en un ámbito donde existen escasos estudios de este tipo. Sin embargo, se considera que serían necesarios estudios adicionales en series de mayor tamaño de pacientes pediátricos que puedan llegar a validar los resultados descritos en el presente trabajo. No obstante, es preciso destacar que la CUN fue uno de los primeros centros en implantar programas de cirugía conservadora de la extremidad, junto con la quimioterapia intraarterial. Dado que esta patología es poco frecuente (su incidencia media se estima en torno a 1 caso/millón de habitantes/año), existen pocos hospitales conocidos con experiencia en este campo. Por este motivo, la obtención de series de mayor tamaño multicéntricas puede suponer un reto.

Otro punto importante a destacar es la gran variabilidad de dosis para cada uno de los procedimientos de angiografía realizados. Esto es debido a que la cantidad de radiación administrada depende en gran medida de la dificultad del mismo y ésta varía en función de la vascularización y la localización del tumor, de las características individuales de cada paciente y de la experiencia del profesional que lo realiza.

No existen dudas acerca de los beneficios de las pruebas de imagen para los pacientes y la necesidad de su realización. De hecho, no se deben olvidar los beneficios que se obtienen de este tratamiento. Con la quimioterapia intraarterial se aumenta el grado de necrosis, por lo que mejora la supervivencia y además permite que en la mayoría de los casos la resección tumoral sea posible sin amputación de la extremidad. Sin embargo, el incremento en el uso de pruebas de radiología intervencionista ha supuesto un gran impacto, no solo sobre el mejor diagnóstico y cuidado del paciente, sino también sobre el hecho de que haya aumentado su exposición a la radiación. Al hablar de los efectos deterministas de la radiación, aunque tiene un umbral, es importante resaltar que la magnitud del efecto sobre el individuo será mayor cuanto mayor es la dosis de radiación ionizante recibida. Por otro lado, los efectos estocásticos son más probables al aumentar la dosis de radiación. En general, el daño causado por la radiación puede ser reparado, por lo que una misma dosis se tolera mejor si se recibe de forma paulatina.

Debido a que el número de procedimientos es un buen predictor de la radiación total que reciben estos pacientes, como se ha demostrado en el presente estudio, será importante optimizar no solo la dosis de cada procedimiento, sino también en lo posible el número de pruebas a las que serán sometidos a lo largo del tratamiento.

Por otro lado, apoyando esta idea, se han propuesto valores de referencia de dosis para diagnóstico (DRL) en procedimientos de radiología intervencionista en España. Estos niveles se establecen como el tercer cuartil de las distribuciones de dosis a pacientes obtenidas en una muestra amplia de centros ${ }^{5,7}$. El valor aportado para los procedimientos de arteriografía de miembros inferiores en un adulto es un $\mathrm{PDA}=69 \mathrm{~Gy} \cdot \mathrm{cm}^{2}$. Sin embargo, ambos procedimientos no son comparables. En una arteriografía de extremidades inferiores se introduce un catéter en la aorta (10 segundos de radioscopia) y se obtienen imágenes del contraste pasando a través de las piernas. En cada sesión de QTia se realizan un mayor número de imágenes, ya que es preciso delimitar la vascularización del tumor e introducir la medicación.

No conviene olvidar que los DRL aportados por estudios previos son para una 
población adulta de referencia, ya que no existen datos en población infantil para angiografía de extremidades inferiores. Además, no existen datos de referencia de radiación en un procedimiento tan específico como es la angiografía para administración de quimioterapia intraarterial. Los datos del presente estudio se centran en una población de niños y adolescentes, que por su superficie corporal, reciben menos dosis de radiación que un adulto. En el presente estudio se observó una disminución del total de la radiación recibida por los pacientes menores de 15 años con respecto al total de pacientes de edades comprendidas entre 15 y menores de 18 años, si bien estas diferencias no llegaron a ser significativas. Los niños y adolescentes son una población especialmente sensible a los efectos de la radiación. Existen evidencias epidemiológicas de que la exposición a radiaciones en niveles moderados puede producir una incidencia incrementada de tumores sólidos, así como de leucemia ${ }^{8}$. El Comité Científico de las Naciones Unidas para el Estudio de los Efectos de las Radiaciones Atómicas determinó que el riesgo de desarrollar cáncer de aquellos sujetos expuestos a radiación ionizante siendo niños, podría ser de 2 a 3 veces superior al de aquellos expuestos a la misma cantidad, pero en edades más avanzadas 9 .

Por último, es importante resaltar que los pacientes afectos de osteosarcoma están sometidos a pruebas adicionales de imagen durante el diagnóstico, tratamiento y seguimiento de su enfermedad, hecho que incrementa la cantidad de radiación ionizante a la que se encuentran expuestos. La mayor tasa de supervivencia de estos pacientes ha aumentado la necesidad de minimizar los efectos a largo plazo de las radiaciones ionizantes. Por este motivo, se considera relevante llevar a cabo un adecuado seguimiento a lo largo de su vida.

\section{BIBLIOGRAFÍA}

1. Khong PL, Ringertz H, Donoghue V, Frush D, Rehani M, Appelgate K, et al. ICRP publication 121: radiological protection in paediatric diagnostic and interventional radiology. Ann ICRP 2013; 42: 1-63.

2. IAEA. Radiation protection in paediatric radiology. Vienna: International Atomic Energy Agency (IAEA) 2012. Report No.: 71.

3. Rodríguez-Galindo C. Osteosarcoma. En: Sierrasesúmaga L, Antillón F, editor. Tratado de Oncología Pediátrica: enfermedades malignas del niño y del adolescente. Madrid: Pearson Prentice Hall; 2006. p. 638-651.

4. J JFFE N. Historical perspective on the introduction and use of chemotherapy for the treatment of osteosarcoma. Adv Exp Med Biol 2014; 804: 1-30.

5. Vano Carruana E, Fernández Soto JM, Sánchez Casanueva RM, Ten Moron JI. Niveles de referencia de dosis en radiología intervencionista. Radiologia 2013; 55 Suppl. 2: 17-24.

6. BAJPAI J, JAFFE N. Perspectives of the role of chemotherapy in the management of osteosarcoma. J Cancer Ther 2012; 3: 1191-1203.

7. Vano E, SÁnchez R, Fernández JM, Gallego JJ, Verdú JF, de Garay MG et al. Patient dose reference levels for interventional radiology: a national approach. Cardiovasc Intervent Radiol 2009; 32: 19-24.

8. UNSCEAR. Summary of low dose-radiation effects on health. New York: United Nations Scientific Committee on the effects of Atomic Radiation; 2010.

9. ICRP. The 2007 Recommendations of the International Commission on Radiological Protection. ICRP publication 103. Ann ICRP. 2007; 37(2-4):1-332. 